\title{
Transcriptional and post-transcriptional regulation of young genes in plants
}

Vivek Kumar Raxwal ${ }^{1,2, *, \#}$, Somya Singh ${ }^{1, *}$, Manu Agarwal $^{1, \#}$ and Karel Riha ${ }^{2, \#}$

${ }^{1}$ Department of Botany, University of Delhi, Delhi-110007, India

${ }^{2}$ Central European Institute of Technology (CEITEC), Masaryk University, Brno, Czech

Republic

* These authors contributed equally

${ }^{\#}$ Corresponding authors

\section{Keywords}

Young genes, Orphan genes, Non-sense mediated RNA decay, Abiotic stress, Evolutionary capacitance, Open chromatin

\begin{abstract}
(150 words)
New genes continuously emerge from non-coding DNA or by diverging from existing genes, but most of them are rapidly lost and only a few become fixed within the population. We hypothesized that young genes are subject to transcriptional and post-transcriptional regulation to limit their expression and minimize their exposure to purifying selection. We found that young genes in rice have relatively low expression levels, which can be attributed to distal enhancers, and closed chromatin conformation at their transcription start sites (TSS). The chromatin in TSS regions can be re-modeled in response to abiotic stress, indicating conditional expression of young genes. Furthermore, transcripts of young genes in Arabidopsis tend to be targeted by nonsense-mediated RNA decay, presenting another layer of regulation limiting their expression. Together, these data suggest that transcriptional and post-transcriptional mechanisms contribute to the conditional expression of young genes, which may alleviate purging selection while providing an opportunity for phenotypic exposure and functionalization.
\end{abstract}




\section{Introduction}

The advent of whole-genome sequencing led to the discovery of a subset of new genes in all domains of life that lack homologs in other lineages (Dujon 1996; Fischer and Eisenberg 1999; Arendsee, et al. 2014; Stein, et al. 2018). These genes, also called orphan or evolutionary young genes, may arise from pre-existing genes by diverging until no homology remains, or they may be born de-novo from non-coding DNA (Tautz and Domazet-Loso 2011; Rodelsperger, et al. 2019; Van Oss and Carvunis 2019). In contrast to old genes, young genes are short, rapidly evolving, and usually do not have essential functions. They are therefore under weaker positive selection (Wolf, et al. 2009; Cai and Petrov 2010). Furthermore, production of nonfunctional proteins from young genes may represent an energetic burden for the cell, and their evolutionarily non-optimized structure can lead to nonproductive interactions, some of which may interfere with cellular functions (Drummond, et al. 2005; Drummond and Wilke 2008). Consequently, despite increasing the population's genetic diversity, most young genes are rapidly lost either due to genetic drift or purging selection, and only a few are fixed in the genome (Palmieri, et al. 2014). This raises the question of whether some young genes have the means to hide from purifying selection, expanding their lifespan in a genome and thereby increasing their chances of acquiring novel functions.

One possibility to mitigate the effect of purifying selection is by limiting the extent of expression and/or conditioning it by developmental or environmental cues. Indeed, young genes often have low expression due to the deposition of repressive chromatin marks and the lack of well-developed cis-regulatory elements required for transcription (Donoghue, et al. 2011; Werner, et al. 2018). The low expression level of young genes can lessen the burden of protein misfolding, hence reducing the negative selection pressure (Drummond, et al. 2005; Drummond and Wilke 2008). Further, spatial or temporal restriction of expression may expand the lifespan of young genes by avoiding untimely exposure to natural selection and provide an opportunity for phenotypic manifestation and functionalization. In nematodes, young genes are born in the vicinity of enhancers and utilize their cis-regulatory elements to express themselves in limited cell-types and tissues (Majic and Payne 2020). Moreover, the permissive transcription environment of isolated compartments, such as the testes in animals and pollen grains in plants, 
provides a perfect breeding ground for young genes to mature and gain function (Vinckenbosch, et al. 2006; Cui, et al. 2015).

In this study, we investigated the mechanisms that contribute to the low or conditional expression of young genes via transcriptional and post-transcriptional regulation in rice and Arabidopsis. We propose that the restricted expression of young genes through these mechanisms can mitigate exposure to negative selection and offer an opportunity for phenotypic manifestation under certain conditions, which permits gene functionalization and genetic fixation in a population.

\section{Result and Discussion}

\section{Young genes are associated with closed chromatin and distal enhancers}

We performed a protein-based homology search across the tree of life to determine the evolutionary age of protein-coding genes present in the rice genome using Phylostratr (Arendsee, et al. 2019). The genes were grouped into phylostrata (PS) according to their evolutionary age such that PS1 contains genes with the oldest known ancestor homolog, whereas the last phylostrata (PS19) includes the evolutionary youngest genes with no known ancestral homolog (Supplementary Figure 1). To understand differences in gene regulation and the underlying reasons, we compared the steady-state expression of evolutionary old (PS1) and young genes (PS19) and observed that young genes largely had low expression relative to the old genes (Figure 1A).

Young genes have been reported to possess heterochromatic epigenetic signatures and a closed chromatin state in Drosophila, nematodes, and Arabidopsis (Li, et al. 2016; Werner, et al. 2018; Zhang and Zhou 2019). To investigate whether the lower expression of young genes in rice was due to the closed chromatin architecture of their regulatory regions, we employed Formaldehyde Assisted Isolation of Regulatory Elements (FAIRE-seq). We observed that the TSS-proximal regions of the young genes have a more closed chromatin conformation relative to old genes (Figure 1B). This is in agreement with higher levels of repressive histone modifications (H3K9me2 and H3K9me1) and lower levels of permissive modifications (H3K9ac and H3K4ac) at the TSS of young genes compared to old genes (Supplementary Figure 2). These results 
suggest that a generally closed chromatin conformation limits the expression of young genes in rice.

Interestingly, our FAIRE-seq analysis in rice revealed an enrichment of open chromatin at the distal intergenic regions (> 1500 bp upstream from TSS) of young genes (Figure 1C), which has also been observed in Arabidopsis (Supplementary Figure 3). Open chromatin at a distal intergenic region is indicative of an enhancer (Zhu, et al. 2015), and enhancers have been suggested to be involved in the birth of young genes (Werner, et al. 2018; Majic and Payne 2020). To determine whether distal upstream regions of young genes indeed contain enhancers, we determined the overlap between open chromatin, which we identified by FAIRE-seq, and enhancers, identified by STARR-seq, in rice (Sun, et al. 2019). Our overlap analysis revealed that more than $60 \%$ of the known enhancers overlapped with open chromatin regions (Figure 1D). Next, we compared the enrichment of enhancers in TSS-proximal and TSS-distal regions. We found that enhancers are enriched in the TSS-distal regions of young genes but not in the TSS-distal regions of old genes (Figure 1E). We also observed that the average age of the nearest gene to distal intergenic enhancers is younger in contrast to the enhancers present in the TSS-proximal region (Figure 1F). Interestingly, we did not detect an enrichment of enhancers in TSS-proximal regions of young genes. This is in contrast to previous findings in nematodes which suggested that genes are born within open regions of enhancers (Werner, et al. 2018). Instead, our result suggests that young genes tend to associate with distally positioned enhancers, possibly because existing cis-regulatory elements are able to restrict their expression (Spitz and Furlong 2012; Andersson, et al. 2014). The utilization of distal enhancers likely contributes to the lower expression observed for young genes, which reduces the cost to the cell of translating misfolded and possibly toxic proteins. Moreover, enhancers evolve faster than promoters, thereby providing an opportunity for young genes to evolve together with enhancers as they acquire novel transcription regulatory networks over time (Prud'homme, et al. 2007; Villar, et al. 2015).

\section{Abiotic stress alters chromatin architecture at TSS of young genes}

Expression of young genes can provide species-specific adaptation to environmental challenges (Donoghue, et al. 2011; Carvunis, et al. 2012), suggesting that their chromatin architecture and transcription are responsive to external stimuli. Since many young genes in rice have a closed 
chromatin conformation in the promoter-proximal regions, we investigated whether abiotic stresses have the potential to affect chromatin conformation. We performed FAIRE-seq on rice seedlings subjected to varying durations of cold, heat, and salt stress (see methods). Except for cold stress (12 h), all of the abiotic stresses we tested increased chromatin accessibility around the TSS of young genes (Figure 2). These results suggest that chromatin architecture can be remodeled upon exposure to external environmental factors, allowing young genes to gradually evolve interactions between cis-regulatory elements and regulatory proteins, thereby providing an opportunity to increase their expression and gain function.

\section{Young gene transcripts are targeted by nonsense-mediated RNA decay}

Apart from transcriptional regulation, the expression of young genes can be reduced by posttranscriptional regulation. Young genes in Drosophila have been associated with increased occurrence of premature translation termination codons (PTC) (Yang, et al. 2015). Since PTCcontaining transcripts are degraded by the non-sense mediated RNA decay (NMD) pathway (Lykke-Andersen and Jensen 2015), we hypothesized that expression of young genes is affected by NMD. To examine this hypothesis, we took advantage of genetic and genomic resources available in Arabidopsis (Raxwal, Simpson, et al. 2020). Indeed, compared to older genes, young genes were enriched for PTCs and long 3`UTRs, both hallmark NMD features (Figure 3a). We also observed an increased incidence of PTCs in young genes of maize and Arabidopsis

(Supplementary Figure 4). We further found that young genes have significantly reduced transcript stability compared to old genes (Figure 3b). The Arabidopsis upfl pad4 mutant, which is severely compromised in NMD (Riehs-Kearnan, et al. 2012; Raxwal, Simpson, et al. 2020), exhibits a more pronounced increase in the expression of young genes than old genes (Figure 3c). These results suggest that young genes are subject to post-transcriptional regulation by NMD.

The increased targeting of young gene mRNAs by NMD implies lower levels of translation. Therefore, we next evaluated the relative efficiency of translation by scoring differences in ribosome association between old and young genes using the Targeted Purification of Polysomal mRNA (TRAP) dataset (Chantarachot, et al. 2020). We observed that transcripts from older genes had substantially higher levels of ribosome association than transcripts from younger genes, indicating less efficient translation of young genes (Figure 3d). Previously, we 
determined that inactivation of NMD leads to increased translation of NMD-targeted transcripts, which was manifested as a shift of the transcripts from monosomal to the polysomal fraction of ribosomes (Raxwal, Simpson, et al. 2020). If NMD contributes to the translational repression of young genes, we also anticipated a similar trend for young gene transcripts. Indeed, NMD inactivation led to a significant decrease of the monosome score for young transcripts, which indicates their shift to polysomes and increased translation (Figure 3E). In contrast, no significant difference was observed in the monosome score of older genes in the presence or absence of UPF1 (Figure 3E). These results further substantiate the role of NMD in limiting expression of young genes at the level of RNA stability and translation. Because NMD efficiency changes in response to environmental and developmental cues (Hug, et al. 2016; Raxwal and Riha 2016), the repression of young genes by NMD is not constitutive. Rather, it could be lifted under certain conditions. The observation that young genes are subject to regulation by NMD is in line with our hypothesis where we proposed that NMD can act as an evolutionary capacitor, permitting accumulation of cryptic genetic variation and expose it conditionally to the selection (Raxwal and Riha 2016).

In conclusion, we propose that young genes tend to have low levels of expression due to closed chromatin, basal transcription by enhancers, and post-transcriptional degradation by NMD to avoid their untimely exposure to purifying selection. All three of these mechanisms are responsive to environmental stress and developmental signals, which provide an opportunity for the expression and phenotypic manifestation of young genes; a prerequisite for gene functionalization.

\section{Methods}

\section{Plant material, growth conditions, and stress treatment}

Oryza sativa L. Japonica nipponbare was grown in a plant growth chamber (Conviron®) at $28^{\circ} \mathrm{C}$ under a photoperiod of $16 \mathrm{~h} / 8 \mathrm{~h}$. 14-day old seedlings were subjected to heat $\left(42^{\circ} \mathrm{C}\right.$ for $30 \mathrm{~min}$ and $3 \mathrm{~h}$ ), cold $\left(4^{\circ} \mathrm{C}\right.$ for $12 \mathrm{~h}$ and $\left.24 \mathrm{~h}\right)$, and salt ( $250 \mathrm{mM} \mathrm{NaCl}$ for $2 \mathrm{~h}$ and $6 \mathrm{~h}$ ) stress. The 14-day old seedlings grown at $28^{\circ} \mathrm{C}$ were taken as the control sample.

\section{Formaldehyde Assisted Isolation of Regulatory Elements (FAIRE)-seq}


FAIRE-seq was performed on two independent replicates of 14-day old stress-treated and control seedlings as per the protocol described previously (Raxwal, Ghosh, et al. 2020). The raw sequencing reads were aligned to the Rice reference genome (IRGSP 1.0) using Bowtie2 with default parameters (Langmead and Salzberg 2012). The reads aligning to the region of the chromosome with a known insertion site of the mitochondrial and chloroplast genome were removed. To remove reads mapped to multiple positions on the genome, reads with a mapping score of less than 10 were filtered out. To remove potential PCR duplicates, reads with the same start and end position were considered only once. Broad peaks were called by MACS 2 with default parameters except the no-model option to identify open chromatin regions in the Rice genome (Zhang, et al. 2008). Further, peaks having read count <1 RPM in any biological replicates were removed from the analysis. A Scatter plot was generated to observe the reproducibility of peaks among biological replicates. The overlap analysis was performed using the $\mathrm{R}$ package ChIPpeakanno (Zhu, et al. 2010).

\section{RNA-seq and analysis}

RNA-seq libraries from RNA extracted (the Qiagen plant RNA extraction kit) from two independent replicates were prepared using the TruSeq RNA sample preparation kit (Illumina Inc., USA). The libraries were sequenced for 50 bp single-end sequencing on Illumina's HiSeq 2000 platform. The sequencing reads were filtered for quality using Trimgalore (https://www.bioinformatics.babraham.ac.uk/projects/trim_galore/) and high-quality reads were pseudo aligned to Rice transcriptome (ensemble 46 version) using Kallisto version 0.46 .0 with default parameters (Bray, et al. 2016). The differential expression analysis was performed using a limma-voom pipeline enabled in 3D-RNA-seq (Guo, et al. 2021).

\section{Evolutionary age classification of genes, NMD features and mRNA half-life analysis}

The evolutionary age or phylostratum of each peptide present in the Rice (Oryza sativa Nipponbare version), Maize (B73 RefGen_v4) and Arabidopsis (Araport 11) was determined using Phylostratr (Arendsee, et al. 2019). A transcript is defined as having an NMD feature if it has premature termination codon (PTC) before (greater than $50 \mathrm{bp}$ ) the last exon junction complex (EJC) or the length of its 3 'UTR exceeds $350 \mathrm{bp}$. The mRNA half-life, TRAP, and NMD data were taken from (Sorenson, et al. 2018; Chantarachot, et al. 2020; Raxwal, Simpson, et al. 2020). 


\section{Acknowledgment}

This work was supported by the Ministry of Education, Youth, and Sports of the Czech Republic, European Regional Development Fund-Project REMAP (grant CZ.02.1.01/0.0/0.0/15_003/0000479 to K.R.), Faculty Research Program Grant from IOE, University of Delhi (grant IoE/2021/12/FRP to MA), India and PURSE grant from the Department of Science and Technology (grant to MA), India. VKR and SS are grateful for research fellowships from the Council of Scientific and Industrial Research (CSIR), India and Department of Biotechnology, India, respectively. We thank Dr Sridhar Sivasubbu and Dr Shamsudheen from the Institute of Genomics and Integrative Biology (IGIB), Delhi, India for helping with the sequencing. 


\section{Figures}

(A)

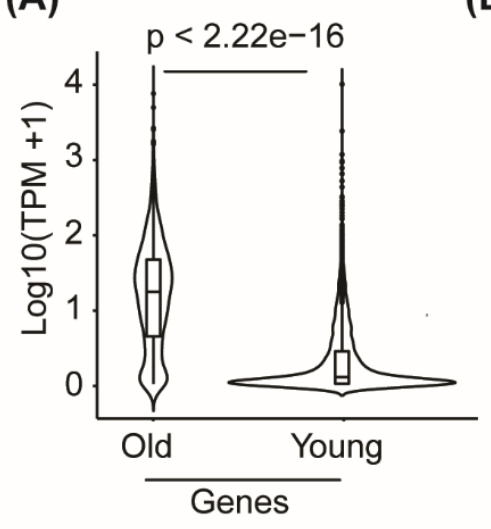

(D)

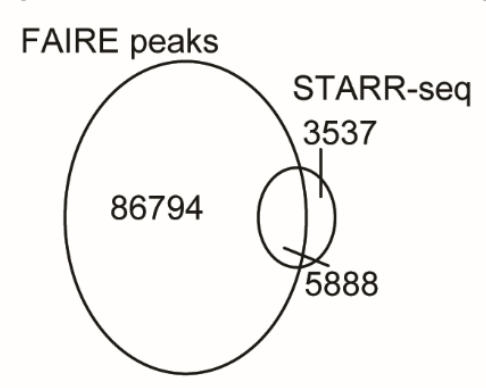

(B)

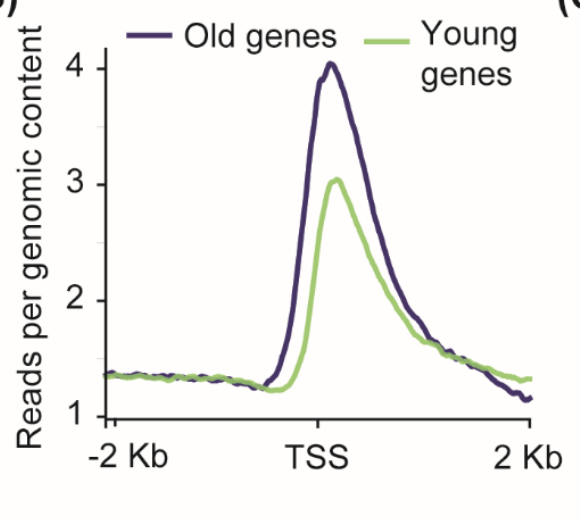

(E)

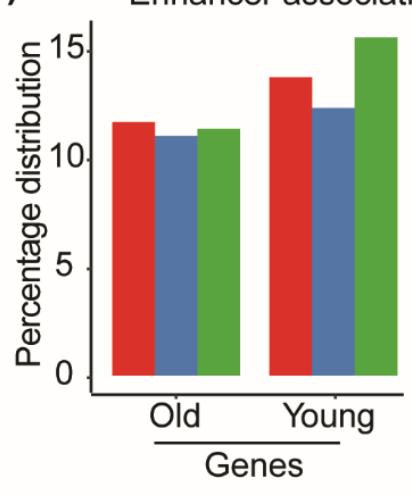

(C)

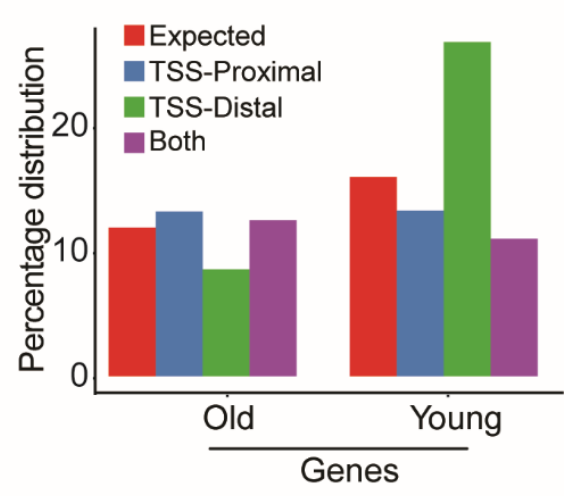

(F)

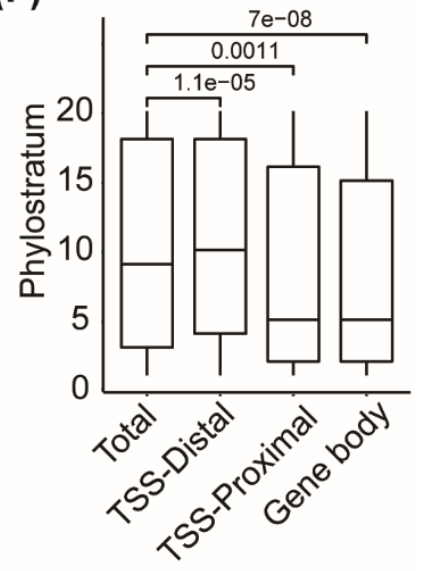

\section{Figure 1: Transcriptional regulation of the young genes.}

(A) A violin plot showing the expression of old and young genes. The expression of genes in TPM (transcript per million) was $\log 10$ transformed before plotting. The statistical significance of the difference was calculated using the Mann-Whitney test. (B) A line plot showing normalized coverage of FAIRE-seq reads around transcription start site (TSS) of old (PS1) and young (PS19) genes. (C) A bar plot was drawn to show the enrichment of FAIRE-seq peaks at either the TSS-proximal (up to $1.5 \mathrm{~kb}$ upstream of TSS), TSS-distal (> -1.5 kb from TSS), or both (FAIRE-seq peak present at TSS-proximal as well as TSS-distal intergenic region) of old and young genes. Enrichment was higher than background (expected), and was calculated as the total percentage of genes present in the old or young gene category. (D) An area proportional 
Venn diagram showing overlap of FAIRE-seq identified open chromatin regions and STARRseq identified enhancers. (E) A bar plot was drawn to show enrichment of enhancers at the TSSproximal (up to $1.5 \mathrm{~kb}$ upstream of TSS) and TSS-distal (>-1.5kb from TSS) regions of old and young genes. The enrichment was seen over the background (expected), calculated as the total percentage of enhancers present in the old or young gene category. (F) A box plot showing the association of STARR-seq identified enhancers with the age of genes. Enhancer is characterized as TSS-Distal if it is present $>1.5 \mathrm{~kb}$ upstream of the TSS, whereas TSS-Proximal enhancers are located within $1.5 \mathrm{~kb}$ upstream of TSS. If an enhancer is present at any part of the gene body, then it is characterized as Gene body enhancer. The age of the nearest gene is plotted at the Yaxis, where phylostratum 1 denotes the oldest and phylostratum 19 denote the youngest class of genes. 


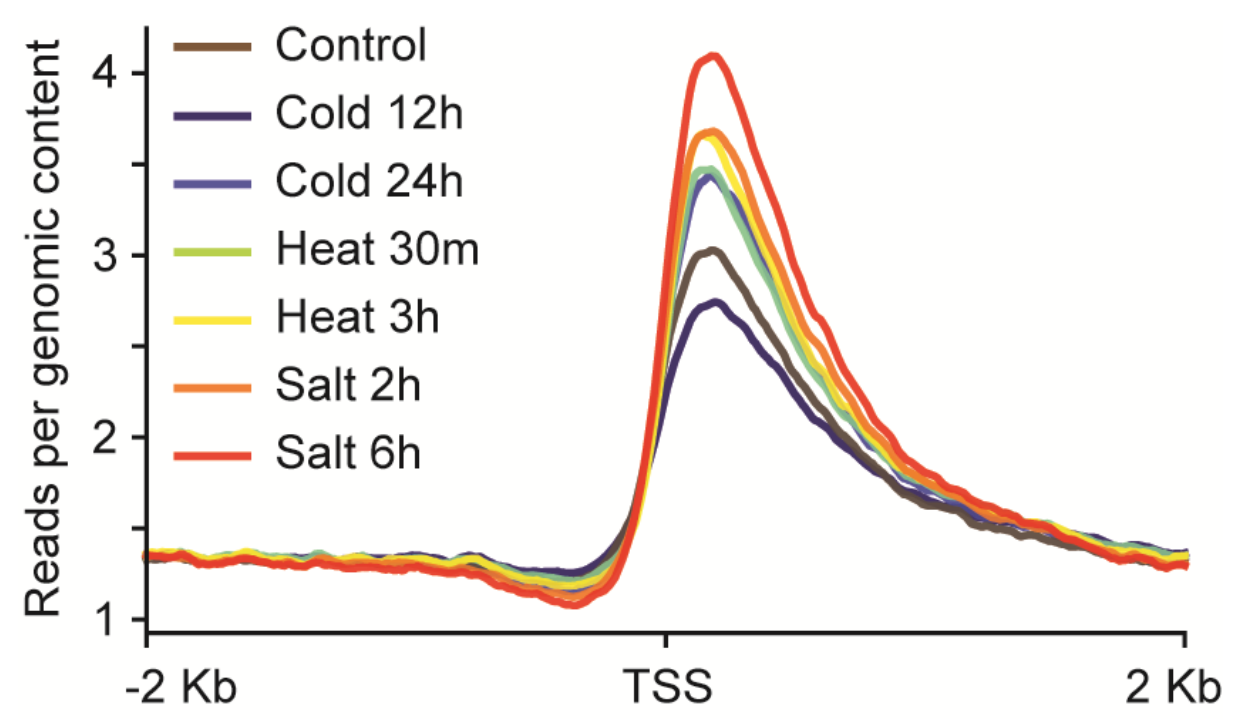

Figure 2: Abiotic stress re-models the chromatin architecture of young genes

Normalized coverage of FAIRE-seq reads around the TSS of young genes plotted as a line plot. 
(A)

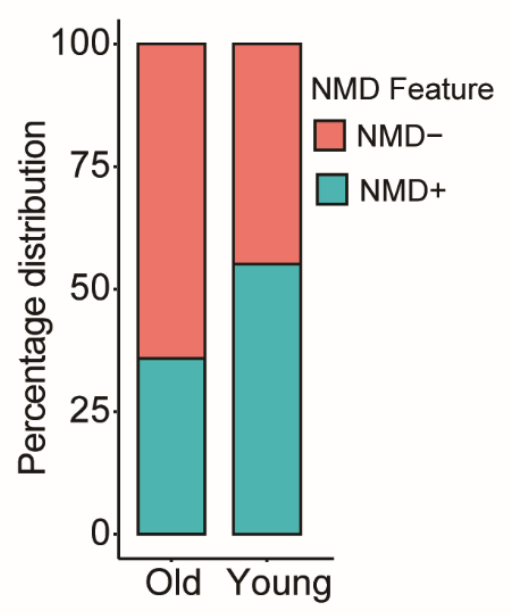

(B)

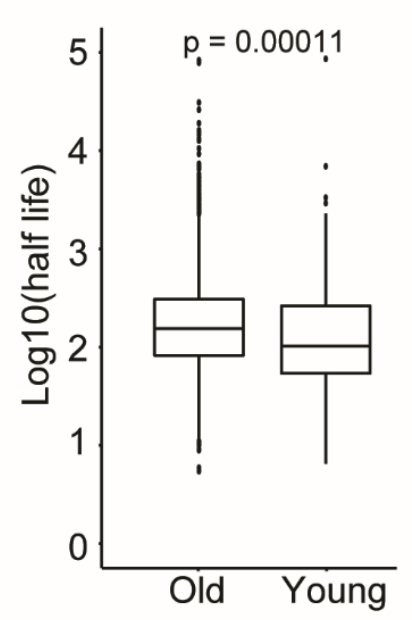

(C)

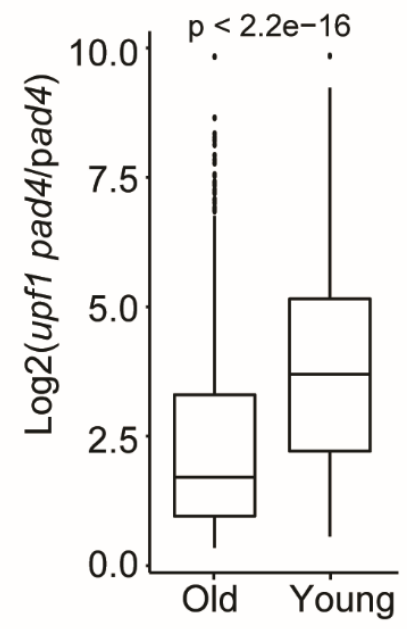

(D)

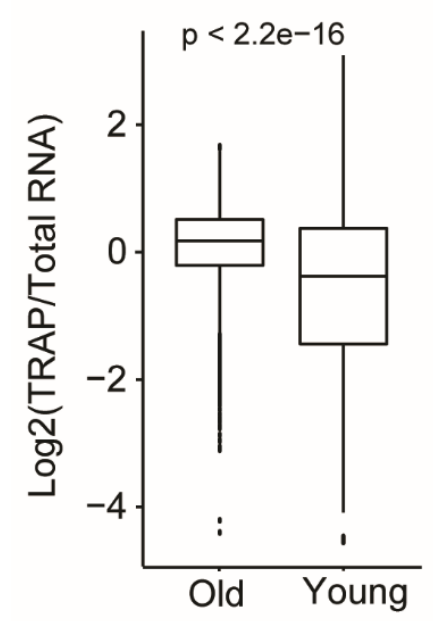

(E)

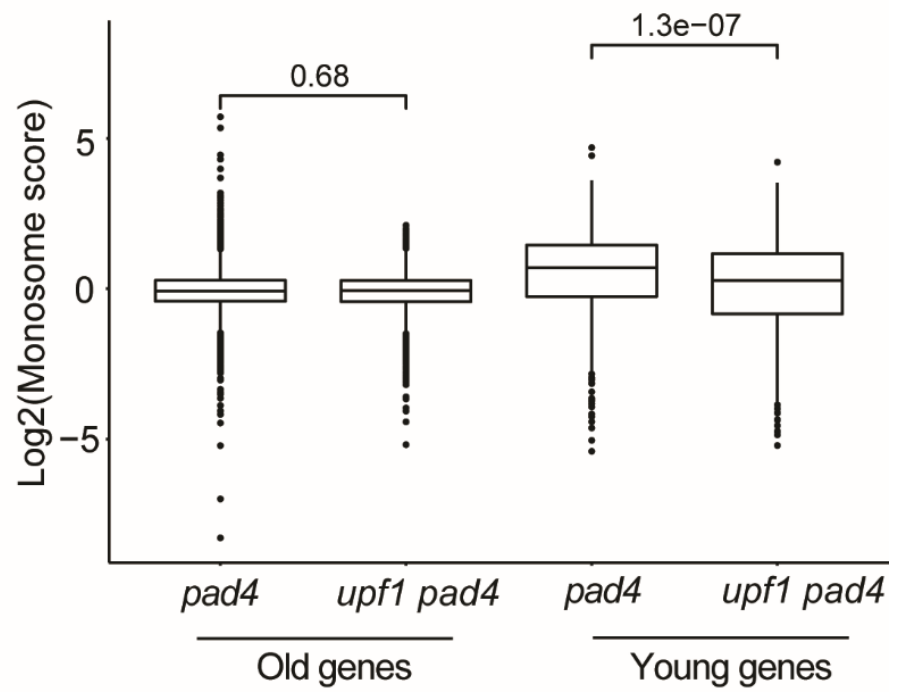

Figure 3: Nonsense-mediated RNA decay (NMD) post-transcriptionally regulates the abundance of young genes.

(A) The accumulation of NMD features in old and young genes is presented as a bar plot. (B) A box plot representing half-life ( $\log 10$ transformed) of the old and young genes. (C) The relative change in expression of old and young genes due to UPF1 deficiency. (D) The association of old and young genes with the ribosome is shown as a box-plot. (E) A box-plot depicting association of old and young genes with monosome and polysome either in pad4 or UPF1-null (upfl pad4). 


\section{Supplementary figures}

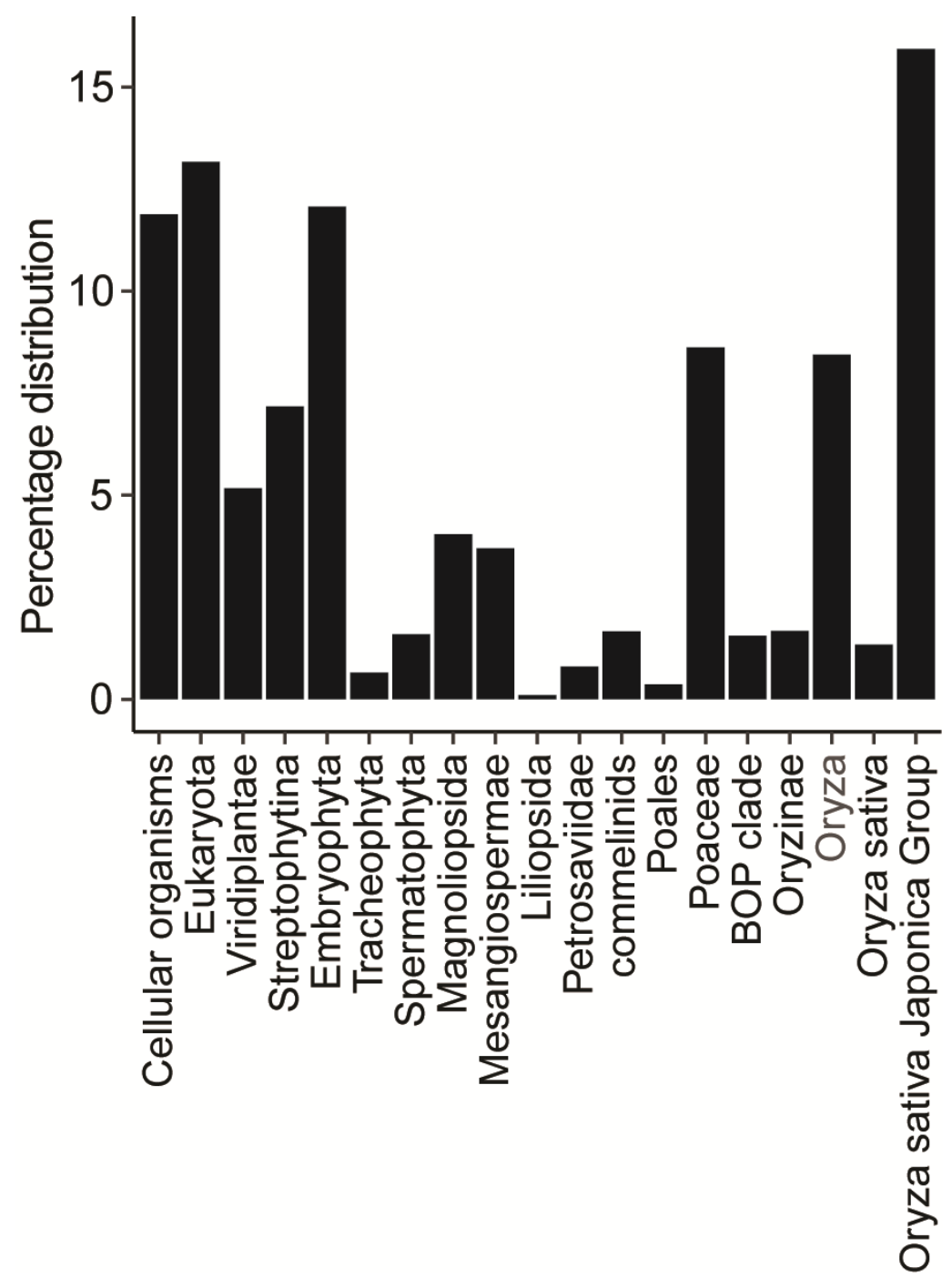

Supplementary Figure 1. Phyllostratographic classification of rice genes showing the distribution of genes with respect to their evolutionary age. 

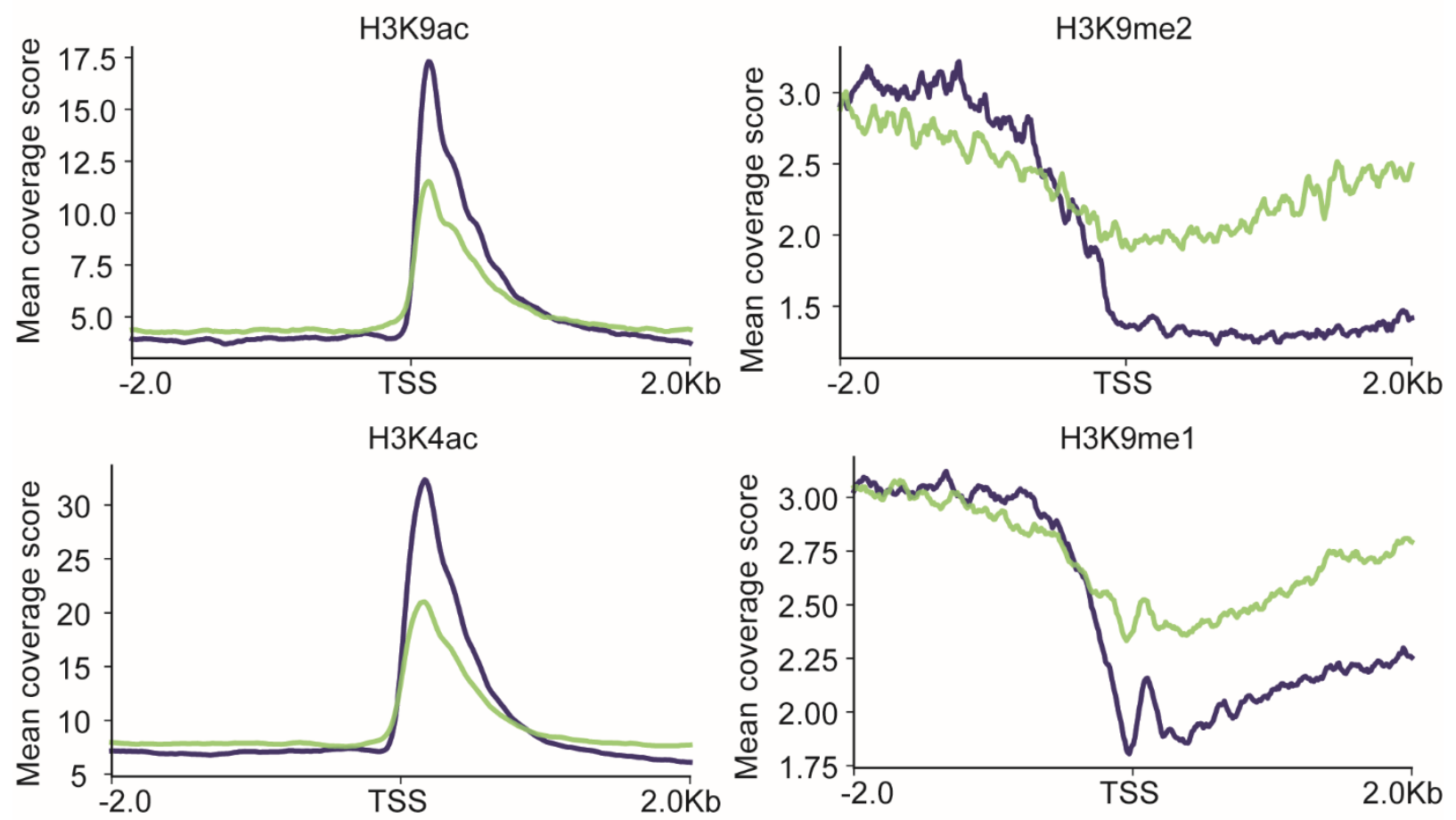

Supplementary Figure 2. A line plot showing the deposition of various histone modification marks over the TSS of young and old genes. 


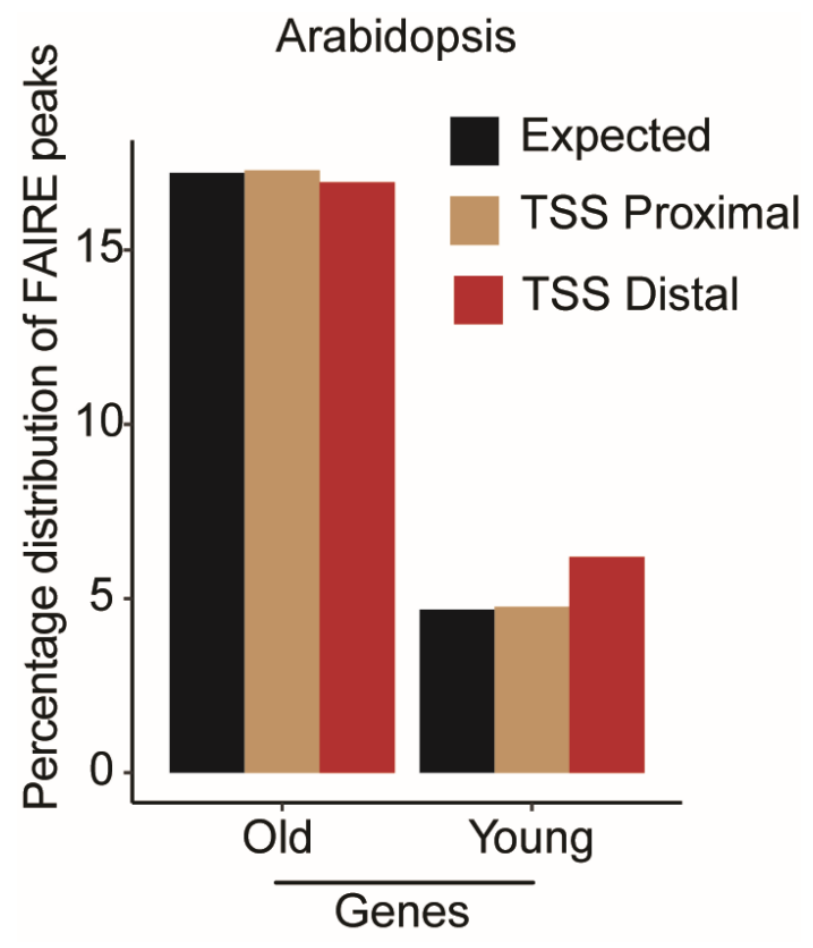

Supplementary Figure 3. A bar plot showing the percentage distribution of FAIRE-peaks in either the TSS proximal (<-1.5kb of TSS) or TSS distal region $(>-1.5 \mathrm{~kb}$ of TSS) of old and young genes of Arabidopsis thaliana. 


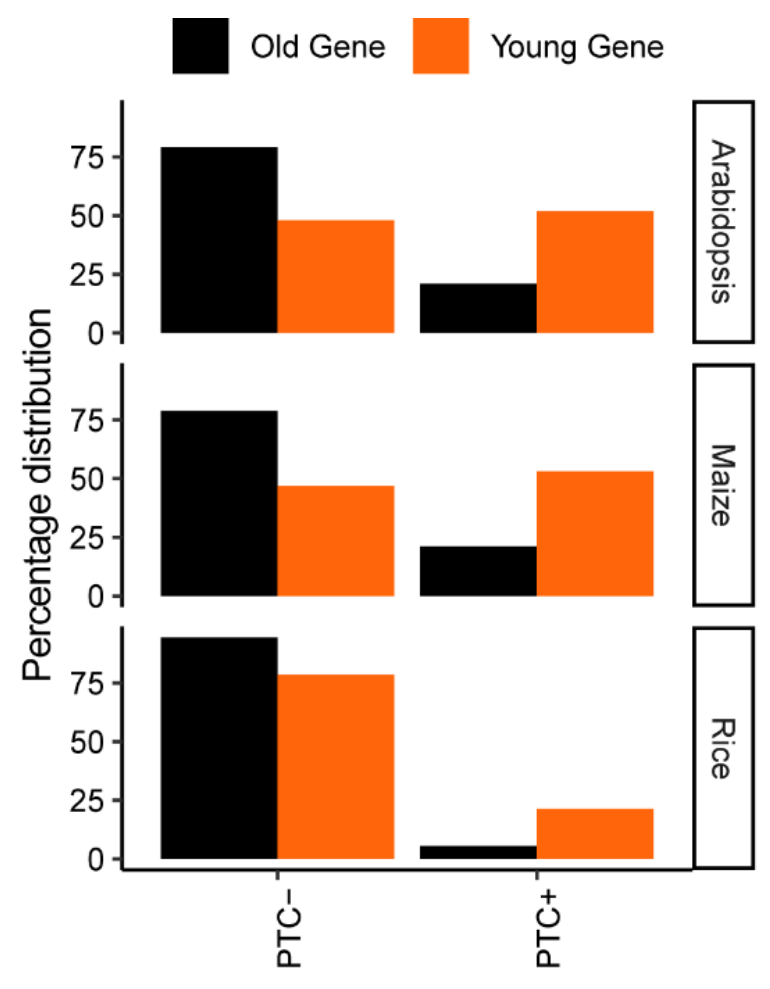

Supplementary Figure 4: A bar plot showing the distribution of premature translation termination codons (PTC) in old and young genes of Arabidopsis, rice, and maize. 


\section{References}

1. Andersson R, Gebhard C, Miguel-Escalada I, Hoof I, Bornholdt J, Boyd M, Chen Y, Zhao X, Schmidl C, Suzuki T, et al. 2014. An atlas of active enhancers across human cell types and tissues. Nature 507:455-+.

2. Arendsee Z, Li J, Singh U, Seetharam A, Dorman K, Wurtele ES. 2019. phylostratr: a framework for phylostratigraphy. Bioinformatics 35:3617-3627.

3. Arendsee ZW, Li L, Wurtele ES. 2014. Coming of age: orphan genes in plants. Trends in Plant Science 19:698-708.

4. Bray NL, Pimentel H, Melsted P, Pachter L. 2016. Near-optimal probabilistic RNA-seq quantification. Nature Biotechnology 34:525-527.

5. Cai JJ, Petrov DA. 2010. Relaxed Purifying Selection and Possibly High Rate of Adaptation in Primate Lineage-Specific Genes. Genome Biology and Evolution 2:393-409.

6. Carvunis AR, Rolland T, Wapinski I, Calderwood MA, Yildirim MA, Simonis N, Charloteaux B, Hidalgo CA, Barbette J, Santhanam B, et al. 2012. Proto-genes and de novo gene birth. Nature 487:370-374.

7. Chantarachot T, Sorenson RS, Hummel M, Ke HY, Kettenburg AT, Chen DN, Aiyetiwa K, Dehesh K, Eulgem T, Sieburth LE, et al. 2020. DHH1/DDX6-like RNA helicases maintain ephemeral half-lives of stress-response mRNAs. Nature Plants 6:675-+.

8. Cui X, Lv Y, Chen ML, Nikoloski Z, Twell D, Zhang DB. 2015. Young Genes out of the Male: An Insight from Evolutionary Age Analysis of the Pollen Transcriptome. Molecular Plant 8:935945.

9. Donoghue MTA, Keshavaiah C, Swamidatta SH, Spillane C. 2011. Evolutionary origins of Brassicaceae specific genes in Arabidopsis thaliana. Bmc Evolutionary Biology 11.

10. Drummond DA, Bloom JD, Adami C, Wilke CO, Arnold FH. 2005. Why highly expressed proteins evolve slowly. Proceedings of the National Academy of Sciences of the United States of America 102:14338-14343.

11. Drummond DA, Wilke CO. 2008. Mistranslation-induced protein misfolding as a dominant constraint on coding-sequence evolution. Cell 134:341-352.

12. Dujon B. 1996. The yeast genome project: what did we learn? Trends in Genetics 12:263-270.

13. Fischer D, Eisenberg D. 1999. Finding families for genomic ORFans. Bioinformatics 15:759-762.

14. Guo WB, Tzioutziou NA, Stephen G, Milne I, Calixto CPG, Waugh R, Brown JWS, Zhang RX. 2021. 3D RNA-seq: a powerful and flexible tool for rapid and accurate differential expression and alternative splicing analysis of RNA-seq data for biologists. Rna Biology 18:1574-1587.

15. Hug N, Longman D, Caceres JF. 2016. Mechanism and regulation of the nonsense-mediated decay pathway. Nucleic Acids Research 44:1483-1495.

16. Langmead B, Salzberg SL. 2012. Fast gapped-read alignment with Bowtie 2. Nature Methods 9:357-U354.

17. Li ZW, Chen X, Wu Q, Hagmann J, Han TS, Zou YP, Ge S, Guo YL. 2016. On the Origin of De Novo Genes in Arabidopsis thaliana Populations. Genome Biology and Evolution 8:2190-2202.

18. Lykke-Andersen S, Jensen TH. 2015. Nonsense-mediated mRNA decay: an intricate machinery that shapes transcriptomes. Nature Reviews Molecular Cell Biology 16:665-677.

19. Majic P, Payne JL. 2020. Enhancers Facilitate the Birth of De Novo Genes and Gene Integration into Regulatory Networks. Molecular Biology and Evolution 37:1165-1178.

20. Palmieri N, Kosiol C, Schlotterer C. 2014. The life cycle of Drosophila orphan genes. Elife 3.

21. Prud'homme B, Gompel N, Carroll SB. 2007. Emerging principles of regulatory evolution. Proceedings of the National Academy of Sciences of the United States of America 104:86058612. 
22. Raxwal VK, Ghosh S, Singh S, Katiyar-Agarwal S, Goel S, Jagannath A, Kumar A, Scaria V, Agarwal M. 2020. Abiotic stress-mediated modulation of the chromatin landscape in Arabidopsis thaliana. J Exp Bot 71:5280-5293.

23. Raxwal VK, Riha K. 2016. Nonsense mediated RNA decay and evolutionary capacitance. Biochimica et Biophysica Acta (BBA)-Gene Regulatory Mechanisms 1859:1538-1543.

24. Raxwal VK, Simpson CG, Gloggnitzer J, Entinze JC, Guo WB, Zhang RX, Brown JWS, Riha K. 2020. Nonsense-Mediated RNA Decay Factor UPF1 Is Critical for Posttranscriptional and Translational Gene Regulation in Arabidopsis. Plant Cell 32:2725-2741.

25. Riehs-Kearnan N, Gloggnitzer J, Dekrout B, Jonak C, Riha K. 2012. Aberrant growth and lethality of Arabidopsis deficient in nonsense-mediated RNA decay factors is caused by autoimmune-like response. Nucleic Acids Research 40:5615-5624.

26. Rodelsperger C, Prabh N, Sommer RJ. 2019. New Gene Origin and Deep Taxon Phylogenomics: Opportunities and Challenges. Trends in Genetics 35:914-922.

27. Sorenson RS, Deshotel MJ, Johnson K, Adler FR, Sieburth LE. 2018. Arabidopsis mRNA decay landscape arises from specialized RNA decay substrates, decapping- mediated feedback, and redundancy. Proceedings of the National Academy of Sciences of the United States of America 115:E1485-E1494.

28. Spitz F, Furlong EEM. 2012. Transcription factors: from enhancer binding to developmental control. Nature Reviews Genetics 13:613-626.

29. Stein JC, Yu Y, Copetti D, Zwickl DJ, Zhang L, Zhang CJ, Chougule K, Gao DY, Iwata A, Goicoechea JL, et al. 2018. Genomes of 13 domesticated and wild rice relatives highlight genetic conservation, turnover and innovation across the genus Oryza. Nature Genetics 50:285-+.

30. Sun J, He N, Niu L, Huang Y, Shen W, Zhang Y, Li L, Hou C. 2019. Global Quantitative Mapping of Enhancers in Rice by STARR-seq. Genomics Proteomics Bioinformatics 17:140-153.

31. Tautz D, Domazet-Loso T. 2011. The evolutionary origin of orphan genes. Nature Reviews Genetics 12:692-702.

32. Van Oss SB, Carvunis AR. 2019. De novo gene birth. Plos Genetics 15.

33. Villar D, Berthelot C, Aldridge S, Rayner TF, Lukk M, Pignatelli M, Park TJ, Deaville R, Erichsen JT, Jasinska AJ, et al. 2015. Enhancer Evolution across 20 Mammalian Species. Cell 160:554-566.

34. Vinckenbosch N, Dupanloup I, Kaessmann H. 2006. Evolutionary fate of retroposed gene copies in the human genome. Proceedings of the National Academy of Sciences of the United States of America 103:3220-3225.

35. Werner MS, Sieriebriennikov B, Prabh N, Loschko T, Lanz C, Sommer RJ. 2018. Young genes have distinct gene structure, epigenetic profiles, and transcriptional regulation. Genome Research 28:1675-1687.

36. Wolf YI, Novichkov PS, Karev GP, Koonin EV, Lipman DJ. 2009. The universal distribution of evolutionary rates of genes and distinct characteristics of eukaryotic genes of different apparent ages. Proceedings of the National Academy of Sciences of the United States of America 106:7273-7280.

37. Yang HW, He BZ, Ma HJ, Tsaur SC, Ma CY, Wu Y, Ting CT, Zhang YE. 2015. Expression Profile and Gene Age Jointly Shaped the Genome-Wide Distribution of Premature Termination Codons in a Drosophila melanogaster Population. Molecular Biology and Evolution 32:216-228.

38. Zhang JY, Zhou Q. 2019. On the Regulatory Evolution of New Genes Throughout Their Life History. Molecular Biology and Evolution 36:15-27.

39. Zhang Y, Liu T, Meyer CA, Eeckhoute J, Johnson DS, Bernstein BE, Nussbaum C, Myers RM, Brown M, Li W, et al. 2008. Model-based Analysis of ChIP-Seq (MACS). Genome Biology 9.

40. Zhu B, Zhang WL, Zhang T, Liu B, Jiang JM. 2015. Genome-Wide Prediction and Validation of Intergenic Enhancers in Arabidopsis Using Open Chromatin Signatures. Plant Cell 27:2415-2426.

41. Zhu LJ, Gazin C, Lawson ND, Pages H, Lin SM, Lapointe DS, Green MR. 2010. ChIPpeakAnno: a Bioconductor package to annotate ChIP-seq and ChIP-chip data. Bmc Bioinformatics 11. 
bioRxiv preprint doi: https://doi.org/10.1101/2021.12.20.473517; this version posted December 21,2021 . The copyright holder for this preprint (which was not certified by peer review) is the author/funder, who has granted bioRxiv a license to display the preprint in perpetuity. It is made available under aCC-BY-NC-ND 4.0 International license. 